\title{
Copeptin for the differential diagnosis and therapy management of hyponatremia in hospitalized patients "The Co-MED-Study"
}

Nicole Nigro, Bettina Winzeler, Isabelle Suter, Birsen Arici, Martina Bally,

Claudine Blum, Philipp Schuetz, Christian Nickel, Roland Bingisser, Andreas Bock, Andreas Huber, Beat Müller, Mirjam Christ-Crain

\section{Background \& Aim}

\section{Background:}

- Hyponatremia is the most common electrolyte disorder in clinical routine and its differential diagnosis is challenging

- The most important osmo- and volume-regulated hormone is arginine vasopressin (AVP). The amount of plasma AVP is regulated by osmotic and non-osmotic stimuli

- The measurement of AVP is cumbersome

- Copeptin is more stable, released in an equimolar ratio with AVP and can be assayed in plasma

Aim of the study:

- To evaluate the usefulness of copeptin as a new diagnostic and prognostic tool in the differential diagnosis and in therapy management of profound hyponatremia
Figure 1: Copeptin, the C-terminal Glycopeptide of the AVP Precursor pre-provasopressin

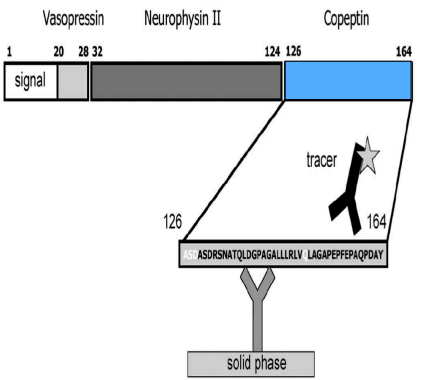

\section{Patients \& Methods}

Setting:

- All patients admitted to the University Hospital of Basel and the Kantonsspital Aarau with profound hyponatremia (Na+ $<125 \mathrm{mmol} / \mathrm{L})$ were included in the study

Work-up on admission:

- Complete medical history

- Clinical items (physical examination, volume status, HR, BP, temp., weight, etc. )

- Routine laboratory tests and copeptin measurements

\section{Final diagnosis:}

- The final diagnosis was made retrospectively by three experts blinded to copeptin levels and was based on our clinical algorithm (see Figure 2), complete chart review and therapy response

Figure 2: Clinical Algorithm

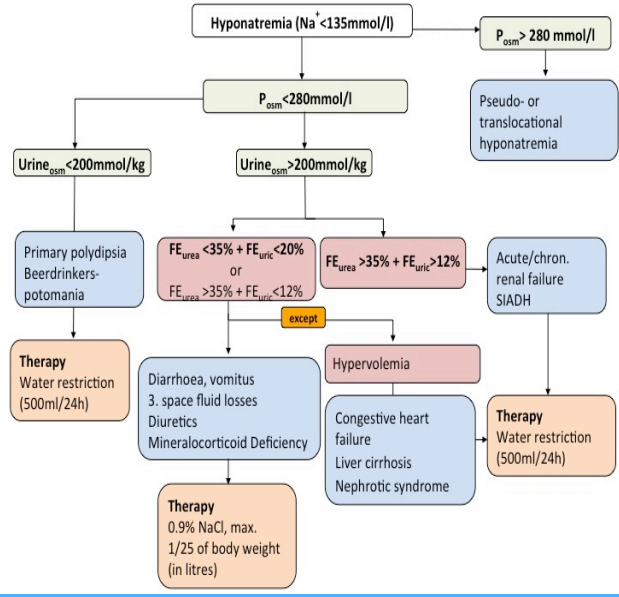

Results

Table 1:Baseline Characteristics at Admission Overall and by Treatment Group

Figure 3: Copeptin and Differential

Figure 4: Copeptin and Treatment Response

Figure 5: ROC Curves to Predict Therapy Diagnosis of Hyponatremia Management

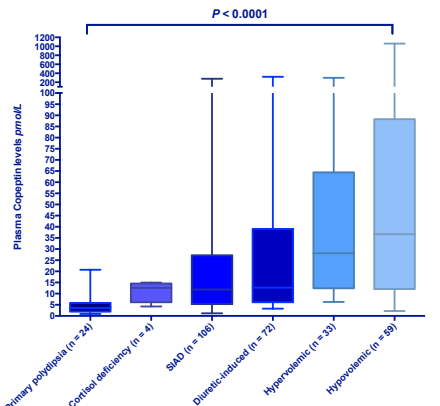

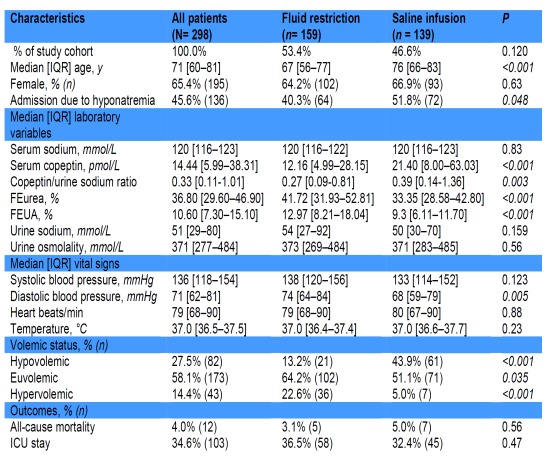

Copeptin levels $<4.4 \mathrm{pmol} / \mathrm{L}$ ( $>56.8 \mathrm{pmol} / \mathrm{L}$ ) predicted fluid restriction (volume administration) with $86 \%(91 \%)$ specificity.
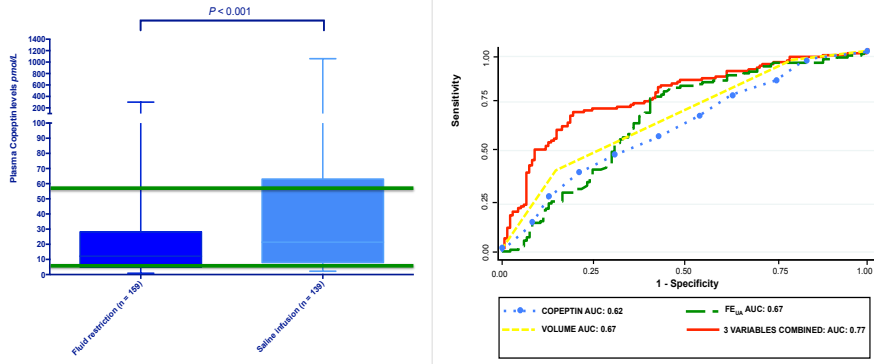

Combining copeptin, volemic status, and $\mathrm{FE}_{\mathrm{UA}}$ improves the prediction for fluid therapy.

\section{Summary \& Conclusion}

Plasma copeptin levels in the differential diagnosis of profund hyponatremia show a wide overlap. Nevertheless, copeptin levels identify a subset of patients with a clear need of saline infusion or fluid restriction and may be a helpful new tool for a more rapid and targeted treatment in patients presenting with profound hyponatremia. The best prediction of therapeutic management is achieved when combining copeptin, volume status and FEuric acid. 